\title{
CORRESPONDENCE COVID-19 outbreak impact on neonatal emergency transport
}

Pediatric Research (2021) 89:1044-1045; https://doi.org/10.1038/s41390020-1027-y

COVID-19 infection is an actual public health challenge. Italy is fighting hard against the virus outbreak. Although infants seem to be spared by the infection, vertical transmission cannot be surely ruled out. Italian neonatologists demonstrated great attention regarding the Neonatal Emergency Transport Service (NETS) involving newborns infected or suspected for COVID-19 infection; in particular, the Neonatal Transport Study Group of the Italian Society of Neonatology (SIN) and a group of neonatologists from the Veneto region recently published recommendation for this highly specific condition ${ }^{1,2}$ by establishing criteria for the safe transport of suspected or confirmed infection in the neonates. During the first days of the crisis, our attention pointed to establish the effective dressing policy for NETS team members, to standardize operational procedures involving spoke hospitals of our network area, and to adapt ambulance and devices, if necessary, as well as to specific COVID-19 transport. ${ }^{1-5}$ We can affirm that at the moment there are no problems in fulfilling these specific active procedures. Internal Gaslini procedures established that each out-born transported newborn must be labeled as COVID-19 positive until a negative swab was available, thus entailing that in the clinical practice each neonatal transport now is a COVID-19-positive transport. The first COVID-19 transport was performed by our NETS on March 18. On May 14, we performed a total of 24 COVID-19 transports. By a retrospective analysis, a previously totally ignored fact emerged clearly. In the figure, the time distribution sequence of a pre-COVID-19 standard transport was drafted. The median time to complete a transport in our regional organization was $\sim 120 \mathrm{~min}$, from the hospital call asking for the transport to the end of the transport, that is, the admission of the transported newborn in Neonatal Intensive Care Unit (NICU). Times of actual COVID-19 transports (Fig. 1) were reviewed. The figure clearly demonstrated that all the newly introduced procedures to perform a safe COVID-19 transport impacted widely on total transport time. Dressing and undressing procedures, and ambulance and device disinfection are the most involved, although the need to obtain further clinical documentation and the difficulty to perform usual clinical stabilization procedures wearing uncomfortable individual protective devices are also involved. As the figure demonstrated, the total median time of a COVID-19 transport redoubled, accounting now at $\sim 240 \mathrm{~min}$. This is a dramatic result. In Italy, the COVID-19 lockdown followed by the introduction of a great number of previously non-expected procedures started at the beginning of March. Although at the moment of writing this letter the Italian Government began to reduce restrictions, it is not predictable when it will be possible to obtain normalcy. Political media affirmed that such a situation could prolong till the end of 2020. Last year our "on-call" NETS performed a total of 312 transports; even excluding about 100 back-transport we performed during 2019, thus calculating $\sim 200$ transport per year, and considering the double time now occurring for a single transport, one must imagine an annual task that in working time might correspond to $\sim 400-450$ transports per year. It is bearable to deal with such a large amount of activity. We believe that it is very important to introduce new and effective procedures to fight the COVID-19 outbreak, but it is likewise, if more, important to evaluate the real impact these procedures produce to avoid to cause further unexpected problems and difficulties.
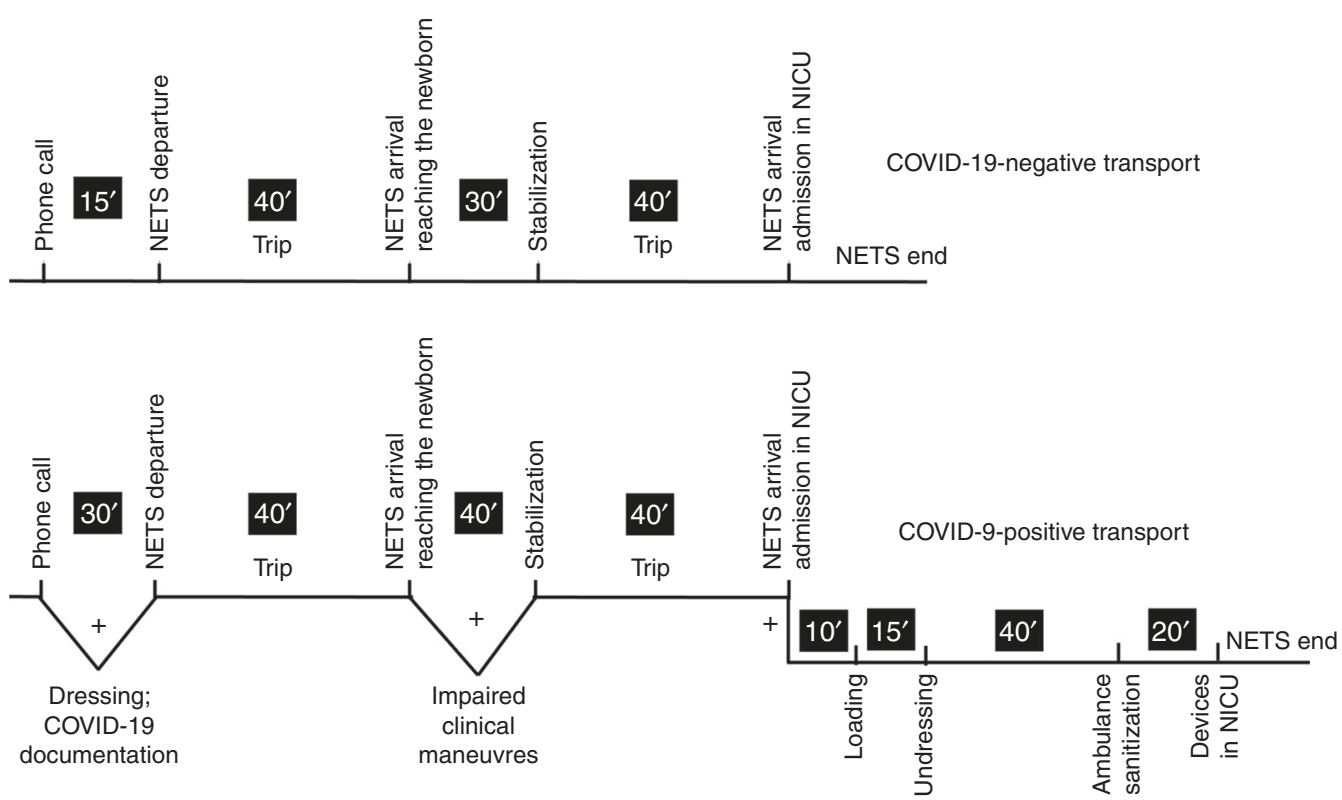

Fig. 1 Impact on neonatal emergency transport time work by COVID-19 outbreak. Different section times for both COVID-19 negative and positive transport are shown in black squares.

Received: 19 May 2020 Accepted: 28 May 2020

Published online: 23 June 2020 


\section{ADDITIONAL INFORMATION}

Competing interests: The author declares no competing interests.

Publisher's note Springer Nature remains neutral with regard to jurisdictional claims in published maps and institutional affiliations.

Carlo Bellini ${ }^{1}$

${ }^{1}$ Neonatal Emergency Transport Service, Neonatal Intensive Care Unit, Department Mother\&Child, IRCCS Gaslini Institute, Genoa, Italy Correspondence: Carlo Bellini (carlobellini@gaslini.org)

\section{REFERENCES}

1. Bellini, C., Gente, M., on behalf of the Neonatal Transport Study Group of the Italian Society of Neonatology. Neonatal transport and COVID-19 outbreak. Air Med. J. https://doi.org/10.1016/j.amj.2020.03.003 (2020).
2. Cavicchiolo, M. E. et al. Neonatal emergency transport system during COVID-19 pandemic in the Veneto Region: proposal for standard operating procedures. Pediatr. Res. https://doi.org/10.1038/s41390-020-0937-z (2020).

3. Maternal and Fetal Experts Committee, Chinese Physician Society of Obstetrics and Gynecology, Chinese Medical Doctor Association, Obstetric Subgroup, Society of Obstetrics and Gynecology, Chinese Medical Association, Society of Perinatal Medicine, Chinese Medical Association, Editorial Board of Chinese. Journal of Perinatal Medicine Proposed management of 2019-novel coronavirus infection during pregnancy and puerperium. Chin. J. Perinat. Med 23, 73-79 (2020).

4. Wang, L., Working Committee on Perinatal and Neonatal Management for the Prevention and Control of the 2019 Novel Coronavirus Infection et al. Chinese Expert Consensus on the Perinatal and Neonatal Management for the Prevention and Control of the 2019 Novel Coronavirus Infection (First Edition). Ann. Transl. Med. 8, 47-55 (2020).

5. Chandrasekharan, P. et al. Neonatal resuscitation and post-resuscitation care of infants born to mothers with suspected or confirmed SARS-CoV-2 infection. Am. J. Perinatol. https://doi.org/10.1055/s-0040-1709688 (2020). 